Provided for non-commercial research and education use. Not for reproduction, distribution or commercial use.

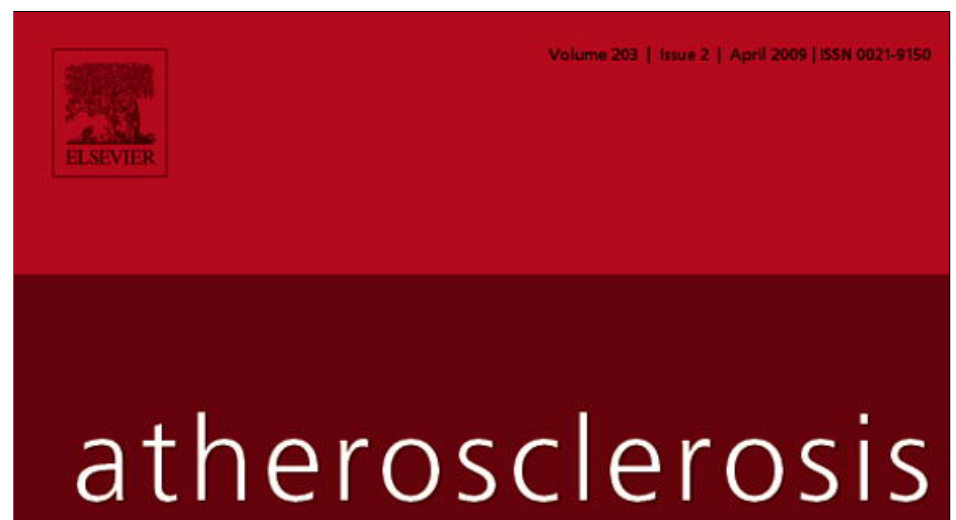

Editor-in-Chief: Professor Steve Humphries

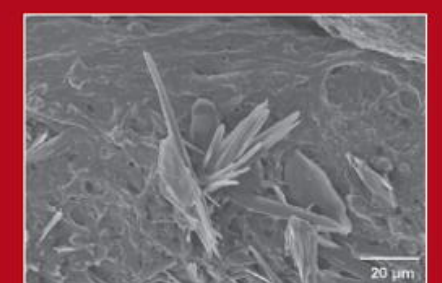

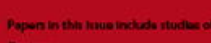

hovener

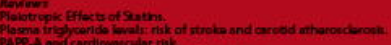

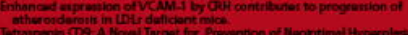

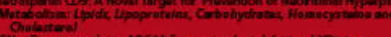

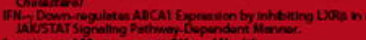

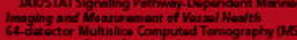

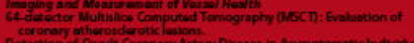

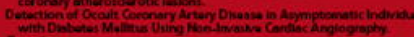

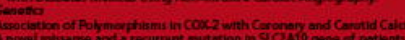

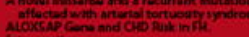

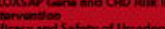

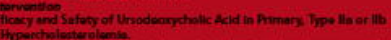

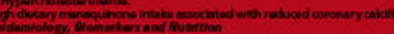

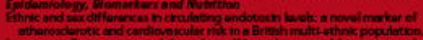

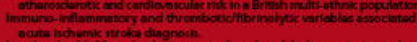

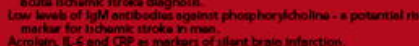

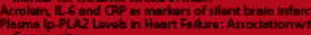

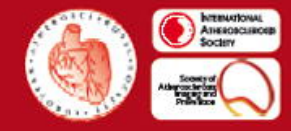

This article appeared in a journal published by Elsevier. The attached copy is furnished to the author for internal non-commercial research and education use, including for instruction at the authors institution and sharing with colleagues.

Other uses, including reproduction and distribution, or selling or licensing copies, or posting to personal, institutional or third party websites are prohibited.

In most cases authors are permitted to post their version of the article (e.g. in Word or Tex form) to their personal website or institutional repository. Authors requiring further information regarding Elsevier's archiving and manuscript policies are encouraged to visit:

http://www.elsevier.com/copyright 


\title{
Immuno-inflammatory and thrombotic/fibrinolytic variables associated with acute ischemic stroke diagnosis
}

\author{
Antonino Tuttolomondo*, Antonio Pinto, Salvatore Corrao, Domenico Di Raimondo, \\ Paola Fernandez, Riccardo Di Sciacca, Valentina Arnao, Giuseppe Licata \\ Dipartimento Biomedico Di Medicina Interna e Specialistica, Università degli Studi di Palermo, Italy
}

\section{A R T I C L E I N F O}

\section{Article history:}

Received 7 February 2008

Received in revised form 20 May 2008

Accepted 26 June 2008

Available online 9 July 2008

\section{Keywords:}

Stroke

Cytokines

PAI-1

TPA

\begin{abstract}
A B S T R A C T
Introduction: Accumulating evidence suggests that inflammation plays an important role in the development of acute cerebrovascular disease. The aim of this study is to evaluate the predictive value of a series of candidate serum immuno-inflammatory and thrombotic/fibrinolitic molecules towards diagnosis of acute ischemic stroke.

Materials and methods: We enrolled 120 consecutive patients with a diagnosis of acute ischemic stroke and 123 consecutive hospitalized control patients without a diagnosis of acute ischemic stroke. We evaluated plasma levels of IL- $1 \beta$, TNF- $\beta$, IL- 6 and IL-10, E-selectin, P-selectin, sICAM- 1 and sVCAM- 1 as markers of immuno-inflammatory activation, vWF plasma levels as a marker of endothelial dysfunction, TPA antigen and PAI-1 plasma levels as a marker of a prothrombotic state.

Results: TNF- $\alpha$, PAI- 1 and TPA on bivariate logistic regression were highly correlated to stroke diagnosis. Among the other variables maintained in the final model IL $\beta$, Selectin E, were significantly associated with acute ischemic stroke diagnosis, whereas IL-6, VICAM-1, ICAM-1 and neutrophil percentage showed only a slight or no association with stroke diagnosis. Furthermore, only the continuous values of TNF- $\alpha$, PAI- 1 and TPA showed a significant predictive value and likelihood ratio, with an area under the ROC curve of $98.6 \%, 97.1 \%$ and $99.9 \%$, respectively.

Discussion: Our findings could suggest the high diagnostic power of these immuno-inflammatory and thrombotic/fibrinolytic variables in patients with acute ischemic stroke. Although our results are encouraging, additional studies are needed to establish the validity of this approach.
\end{abstract}

@ 2008 Elsevier Ireland Ltd. All rights reserved.

\section{Introduction}

Accumulating evidence suggests that inflammation plays an important role in the development of cardiovascular and cerebrovascular disease [1,2]. Over the past few years has been stressed the role of inflammation in the pathophysiology of acute brain ischemia and how most inflammatory events are mediated by cytokines, small glycoproteins expressed by many cell types in response to acute cerebral ischemia [3,4]. Recently our group [5] showed that stroke patients, compared to controls, have significantly higher median plasma levels of some cytokines, adhesion molecules and selectins until $72 \mathrm{~h}$ after onset of symptoms. The central role of inflammation, expressed by cytokine cascade in the pathogenesis of neuronal ischemic damage, could suggest

\footnotetext{
* Corresponding author at: Dipartimento Biomedico di Medicina Interna e Specialistica, Università degli Studi di Palermo, P.zza delle Cliniche n.2, 90127 Palermo, Italy. Tel.: +39 091 6552128; fax: +39 0916552285 .

E-mail address: brunotutto@unipa.it (A. Tuttolomondo).
}

a possible diagnostic role of candidate serum markers of this immuno-inflammatory activation. In fact, the absence of widely available and sensitive diagnostic tests for acute cerebral ischemia remain, at present, a problem in the diagnosis and management of stroke. Unlike acute coronary syndromes and congestive heart failure with the myocardial isoform of creatine phosphokinase, troponin and B-type natriuretic peptide, there is no single biochemical diagnostic marker available for ischemic stroke that is sufficiently sensitive and specific. To date, most clinical studies concerning the evaluation of the immuno-inflammatory profile of patients with ischemic stroke have been limited by low numbers of patients enrolled and by the fact that no single biochemical marker has been demonstrated to possess the requisite sensitivity and specificity to allow it to be used as a diagnostic marker. Recently Lynch et al. [6] examined the feasibility of developing a diagnostic panel of biochemical markers of cerebral ischemia, and reported that a panel of biochemical markers comprising $S 100 \beta$, matrix metalloproteinase-9, vascular cell adhesion molecule and von Willebrand factor may be helpful in identifying stroke patients in the prehospital setting. 
Taking into account the complexity of the brain ischemic cascade mechanisms involving immuno-inflammatory, thrombotic and fibrinolytic pathways, the aim of this study is to evaluate the predictive value of some candidate serum immuno-inflammatory and thrombotic/fibrinolytic molecules for acute ischemic stroke diagnosis.

\section{Materials and methods}

We enrolled 120 consecutive patients with a diagnosis of acute ischemic stroke admitted to the Internal Medicine Department at the University of Palermo between November 2002 and January 2005, and 123 hospitalized control patients without a diagnosis of acute ischemic stroke. Control subjects were patients admitted, in the same period, to our Internal Medicine Department for any cause other than acute cardiovascular and cerebrovascular events or exclusion criteria (see below).

Stroke was defined by focal neurological signs or symptoms thought to be of vascular origin that persisted for $>24 \mathrm{~h}$ confirmed by brain CT and/or MRI in baseline conditions and brain CT with contrast medium after 48-72 $\mathrm{h}$ [7].

In order to match patients with acute ischemic stroke and controls also for cardiovascular risk and previous cardiovascular morbidity, controls were included if they had vascular risk factors or a history of myocardial infarction or cerebrovascular disease or peripheral vascular disease, but they were excluded if they had either current or recent (until 6 months) cerebrovascular disease or one of the exclusion criteria (see above).

Cardiovascular risk factors were evaluated for both cases and controls on the basis of the criteria shown below. Hypercholesterolemia was defined as the presence of total cholesterol blood levels $\geq 200 \mathrm{mg} / \mathrm{dL}$. Hypertension was defined as present if subjects had been previously diagnosed according the World Health Organization/International Society of Hypertension guidelines and were routinely receiving antihypertensive therapy. Patients were defined as type 2 diabetics if they had known diabetes treated by diet, oral hypoglycaemic drugs or insulin before stroke Previous coronary artery disease was determined on the basis of a history of physician-diagnosed angina, myocardial infarction, or any previous revascularization procedure assessed by a questionnaire.

Previous cerebrovascular disease (TIA/ischemic stroke) was assessed by history, specific neurologic examination executed by specialists, and hospital or radiological (brain computed tomography or brain magnetic resonance) records of definite previous stroke.

Subjects were classified as having previous peripheral artery disease (PAD) when they had a history of $A B I<0.9$ and/or of intermittens claudicatio or of critical limb ischemia or when they had undergone a peripheral arterial bypass or amputation.

The study protocol was approved by the local ethics committee, and all participants gave written informed consent.

Every subject with ischemic stroke was matched for age $( \pm 3$ years), sex, and cardiovascular risk factor prevalence and previous vascular events with one control subject.

Patients with inflammatory or infectious diseases, cancer, hematological diseases and severe renal or liver failure, as well as those who were under treatment with anti-inflammatory drugs, were excluded.

The type of acute ischemic stroke was classified according to the TOAST classification [6]: (1) large artery atherosclerosis (LAAS); (2) cardioembolic infarct (CEI); (3) lacunar infarct (LAC); (4) stroke of other determined etiology (ODE); (5) stroke of undetermined etiology (UDE). All the ischemic stroke patients underwent: medical history with recording of potential stroke risk factors, blood and coagulation tests, 12-lead ECG, 24 h electrocardiography monitor- ing, transthoracic echocardiography, carotid ultrasound, brain CT or MRI at admission.

Blood samples were obtained in the non-fasting state. After $10 \mathrm{~min}$ of rest in the supine position, $35 \mathrm{~mL}$ blood was collected from the antecubital vein. EDTA-anticoagulated peripheral blood was drawn from each patient within $72 \mathrm{~h}$ of onset of symptoms. Serum and plasma were immediately separated by centrifugation and stored in aliquots at $-80^{\circ} \mathrm{C}$ until analysis.

We evaluated plasma levels of IL-1 $\beta$, TNF- $\alpha$, IL-6 and IL10, E-selectin, P-selectin, sICAM-1 and sVCAM-1 as markers of immuno-inflammatory activation, vWF plasma levels as a marker of endothelial dysfunction, TPA antigen and PAI-1 plasma levels as a marker of a thrombotic/fibrinolytic state.

We chose to evaluate these biomarkers because acute ischemic stroke has been associated with serum elevations of a series of immuno-inflammatory variables such as TNF- $\alpha$, interleukin-6 (IL6 ) and other cytokines, selectins and adhesion molecules [7-10] and of markers of impaired hemostasis and thrombosis [11-13].

IL- $1 \beta$, TNF- $\alpha$, IL- 6 and IL-10 and vWF antigen were measured using a sandwich ELISA (Human IL-1 $\beta$, TNF- $\alpha$, IL-6 and IL-10 Quantikine, R\&D Systems (VWF ELISA kitdurian, Instrumentation Laboratory, Milano, Italy); VCAM-1, ICAM-1, E-selectin, P-selectin, PAI-1 and TPA-antigen were measured by commercial bioimmunoassay (Human sICAM-1, sVCAM-I, sE-selectin and sP-selectin Parameter, Quantikine, R\&D Systems, Gentaur AssayMax Human Plasminogen Activator Inhibitor-1 (PAI-1) ELISA Kit, Gentaur AssayMax Tissue Plasminogen Activator (TPA) ELISA Kit).

The minimum detectable concentrations for the diagnostic tests are: TNF alpha: $1.6 \mathrm{pg} / \mathrm{mL}$, IL- $1 \beta:<1 \mathrm{pg} / \mathrm{mL}$, IL-6: $<0.70 \mathrm{pg} / \mathrm{mL}$, IL-10: $>3.9 \mathrm{pg} / \mathrm{mL}$, ICAM-1: $<0.35 \mathrm{ng} / \mathrm{mL}$, VCAM-1: $0.6 \mathrm{ng} / \mathrm{mL}$, E-Selectin: $<0.1 \mathrm{ng} / \mathrm{mL}$, P-Selectin: $<0.5 \mathrm{ng} / \mathrm{mL}$, vWF: $1.0 \%$, TPA: $0.3 \mathrm{pg} / \mathrm{mL}, \mathrm{PAI}-$ $1:<50 \mathrm{pg} / \mathrm{mL}$.

Intraassay and interassay coefficients of variation were: TNF alpha: $4.2 \%$ and $4.6 \%$; IL-1 $\beta$ : $3.3 \%$ and $4.2 \%$; IL-6: $1.6 \%$ and $3.3 \%$; IL-10: $4.3 \%$ and $7.5 \%$; ICAM-1: $4.8 \%$ and $6.1 \%$; VCAM-1: $3.5 \%$ and 7.7\%; E-Selectin: $4.8 \%$ and 5.7\%; P-Selectin: $4.9 \%$ and $8.8 \%$; vWF: $5 \%$ and $10 \%$; TPA: $4.8 \%$ and $5 \%$; PAI-1: $5.7 \%$ and $8.3 \%$.

\section{Statistical analysis}

Data are reported as median (lower quartile $\leftrightarrow$ upper quartile). Comparisons between groups were performed by Mann-Whitney U-test. The Kruskal-Wallis test was performed as non-parametric analysis of variance for multiple comparisons, and the Conover-Inman procedure was used as post hoc test to make all possible pair-wise comparisons between groups. The Wilcoxon signed ranks test statistic was performed to compare control to baseline data. Pearson chi-square was computed for categorical variables. A two-tailed $p$ value $<0.5$ was considered statistically significant. To investigate relationships between predictor variables and stroke, in the whole study sample, logistic regression technique was performed and stroke was considered the outcome variable. First, a bivariate logistic regression analysis was performed to individuate relationships between predictors and the outcome variable (Stroke). TNF- $\alpha$, TPA and PAI-1 are presented only as bivariate analysis by a high grade of correlation with the outcome variable. These variables were excluded a priori from the final model. TNF- $\alpha$ was transformed into log (base 10) values to run logistic regression analysis. Then, variable selections for the final model and linear relationships between outcome and continuous predictors were investigated as suggested by Hosmer and Lemeshow [14]. Odds ratios and their 95\% confidence intervals were computed. A bootstrap procedure may be used to cross-validate confidence intervals calculated for odds ratios 
Table 1

General and clinical characteristics of stroke patients and controls

\begin{tabular}{|c|c|c|c|}
\hline Variable & Stroke pts ( $n: 120)$ & Controls ( $n: 123)$ & $p$ \\
\hline Age (years) & $72(64-82.5)$ & $69(65-83)$ & 0.749 \\
\hline Glucose blood levels (mg/dL) & $155.5(99-233)$ & $139(87.5-193.5)$ & 0.001 \\
\hline Cholesterol blood levels (mg/dL) & $232(199-260)$ & $200(178-225)$ & $<0.001$ \\
\hline Triglycerides blood levels & $187.5(140.75-211.75)$ & $154(98-200)$ & 0.007 \\
\hline White body cells (WBC) (per $\mathrm{mm}^{3}$ ) & $8000(6700-10,000)$ & $6400(5500-8800)$ & $<0.001$ \\
\hline Neutrophils (\%) & $5780(4071-8000)$ & $3843(336-6320)$ & $<0.001$ \\
\hline $\operatorname{Sex}(M / F)$ & $68 / 52$ & $55 / 68$ & 0.062 \\
\hline Diabetes $(n / \%)$ & $50(41.7)$ & $59(48 \%)$ & 0.363 \\
\hline Hypertension $(n / \%)$ & $74(61.7 \%)$ & $63(51.2 \%)$ & 0.101 \\
\hline Previous stroke $(n / \%)$ & $39(32.5)$ & $37(30.08)$ & 0.75 \\
\hline $\operatorname{CAD}(n / \%)$ & $25(20.8)$ & $24(19.5)$ & 0.46 \\
\hline $\operatorname{PAD}(n / \%)$ & $14(11.6)$ & $17(13.8)$ & 0.23 \\
\hline
\end{tabular}

Data are expressed as median and interquartile (lower and upper quartile). Demographic and anamnestic data are expressed as no. (percentage).

Table 2

Immuno-inflammatory and thrombotic/phybrinolytic variables in stroke subjects vs. controls

\begin{tabular}{|c|c|c|c|}
\hline Variable & Stroke patients & Controls & $p$ \\
\hline$n$ & 120 & 123 & \\
\hline IL-1 $\beta(\mathrm{pg} / \mathrm{mL})$ & $9(4-11)$ & $3(2-5)$ & $<0.001$ \\
\hline IL-6 $(\mathrm{pg} / \mathrm{mL})$ & $11(6-30)$ & $9(2.9-18)$ & $<0.001$ \\
\hline $\mathrm{TNF}-\alpha(\mathrm{pg} / \mathrm{mL})$ & $31.5(10.25-41)$ & $3.7(1.1-4.3)$ & $<0.001$ \\
\hline E-selectin $\mathrm{n} / \mathrm{mL}$ ) & $2.25(2.0-4.0)$ & $2(1-2)$ & $<0.001$ \\
\hline P-selectin (ng/mL) & $4(2-6.3)$ & $3,1(2.1-4)$ & 0.004 \\
\hline $\operatorname{VICAM}(\mathrm{ng} / \mathrm{mL})$ & $20(15.1-23)$ & $14(13-17)$ & $<0.001$ \\
\hline ICAM (ng/mL) & $20.8(16.2-24)$ & $15.9(12-18.1)$ & $<0.001$ \\
\hline $\mathrm{IL}-10(\mathrm{pg} / \mathrm{mL})$ & $3.75(2-9)$ & $4(2-10)$ & 0.433 \\
\hline $\mathrm{vWF}(\mathrm{ng} / \mathrm{mL})$ & $10(6-10)$ & $4(3-8)$ & 0.0001 \\
\hline PAI-1 $(\mathrm{pg} / \mathrm{mL})$ & $142(109.5-175)$ & $21(12-26)$ & $<0.001$ \\
\hline $\mathrm{TPA}(\mathrm{pg} / \mathrm{mL})$ & $24(15.75-36)$ & $74(59-98)$ & $<0.001$ \\
\hline
\end{tabular}

Data are expressed as median and interquartile (lower and upper quartile). Demographic and anamnestic data are expressed as no. (percentage). TNF- $\alpha$ : tumor necrosis

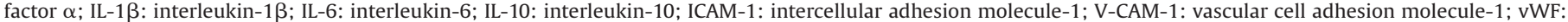
von Willebrand factor; TPA: tissue plasminogen activator; PAI-1: plasminogen activator inhibitor-1.

derived from fitted logistic models [15]. The bootstrap confidence intervals used are the 'bias-corrected' type. To evaluate the predictive role of different values of some immuno-inflammatory variables, we evaluated sensibility, specificity, likelihood ratio and receiver-operating characteristics (ROC) with their area under the curve (AUC) were constructed.

According to sample size calculation, 110 enrolled subjects were needed to detect a minimal relative prognostic risk of 2.1 for patients with different levels of cytokines and thrombotic-fibrinolytic markers with an alfa error of 0.05 and a power of 0.80 .

\section{Results}

General characteristics of patients enrolled in the study are reported in Table 1 . Immuno-inflammatory and thrombotic-fibrinolytic variables are reported in Table 2 .

Among stroke patients, according to TOAST Classification, 50 were classified as LAAS, 46 as lacunar, 20 as CEI and 4 as ODE (see Table 3).

Immuno-inflammatory variables of patients with acute ischemic stroke and of controls are shown in Table 2. Stroke patients compared to controls showed significantly higher median

Table 3

Immuno-inflammatory markers in patients with ischemic stroke in relation of TOAST subtypes

\begin{tabular}{|c|c|c|c|c|c|}
\hline Variable & LAAS & Lacunar & CEI & ODE & $p$ \\
\hline$n$ & 50 & 46 & 20 & 4 & \\
\hline TNF- $\alpha(\mathrm{pg} / \mathrm{mL})$ & $27.5(13.4-40.5)$ & $19.4(9-23)$ & $38.5(22.2-46)$ & $29(10.4-39.0)$ & $<0.0001$ \\
\hline IL-6 $(\mathrm{pg} / \mathrm{mL})$ & $8(4-12)$ & $4(2-9)$ & $11(5,5-19)$ & $7(4.5-12.5)$ & 0.0029 \\
\hline IL-1 $\beta(p g / m L)$ & $9(5-10.5)$ & $6(10-3)$ & $11.5(8-13)$ & $3.5(2-7)$ & $<0.0001$ \\
\hline ICAM-1 (ng/mL) & $20.8(15.9-24)$ & $19.91(15-23)$ & $19.52(18.55-23)$ & $17.25(14.95-18.8)$ & 0.56 \\
\hline VCAM-1 (pg/mL) & $20(15-24)$ & $17(20-15)$ & $20(15.7-26)$ & $17.5(14-19)$ & 0.53 \\
\hline E-selectin (ng/mL) & $3(2-4)$ & $2(2-4)$ & $2.75(2-3.5)$ & $2.5(1.3-3)$ & 0.801 \\
\hline P-selectin (ng/mL) & $4.95(2-6.5)$ & $4(2.4-6)$ & $3,4(1.46-6.43)$ & $1.75(1.05-5.2)$ & 0.49 \\
\hline $\operatorname{vWF}(\mathrm{ng} / \mathrm{mL})$ & $8(6-10)$ & $6(3-8)$ & $10(5-12)$ & $5(4-9)$ & 0.05 \\
\hline PAI-1 (pg/mL) & $134(98-168)$ & $155(108.25-172)$ & $162(123-187)$ & $122(111-187)$ & 0.61 \\
\hline TPA (pg/mL) & $24(14-36)$ & $28(20-38)$ & $22(17-23)$ & $20(18-27)$ & 0.85 \\
\hline
\end{tabular}

Data are expressed as median and interquartile (lower and upper quartile). TNF- $\alpha$ : tumor necrosis factor $\alpha$; IL-1 $\beta$ : interleukin-1 $\beta$; IL-6: interleukin-6; IL-10: interleukin-10; ICAM-1: intercellular adhesion molecule-1; V-CAM-1: vascular cell adhesion molecule-1; vWF: von Willebrand factor; TPA: tissue plasminogen activator; PAI-1: plasminogen activator. 
Table 4

Infarct site at neuroimaging findings (brain CT or MRI) in relation of TOAST diagnostic subtype

\begin{tabular}{|c|c|c|c|}
\hline & Large artery atherosclerosis $(n: 50)$ & Cardioembolic strokes ( $n: 20)$ & Lacunar strokes $(n: 46)$ \\
\hline \multicolumn{4}{|l|}{ Neuroimaging findings } \\
\hline Cortical & $31(62 \%)$ & $16(80 \%)$ & $8(17.3 \%)$ \\
\hline Subcortical & $19(38 \%)$ & $4(20 \%)$ & $28(60.8 \%)$ \\
\hline Internal capsula & $10(52.6 \%)$ & $4(100 \%)$ & $10(35.7 \%)$ \\
\hline Thalamus & $4(21 \%)$ & 0 & $7(25 \%)$ \\
\hline Basal ganglia & $5(26.3 \%)$ & 0 & $5(21.7 \%)$ \\
\hline Pons & 0 & 0 & $2(7.1 \%)$ \\
\hline Corona radiata & 0 & 0 & $4(14.2 \%)$ \\
\hline No lesion CT or MRI detectable & 0 & 0 & $10(21 \%)$ \\
\hline
\end{tabular}

\section{Table 5}

Bivariate logistic regression about relationships between predictor variables and outcome variable (stroke)

\begin{tabular}{lrc}
\hline Variable & $\beta$ & $p$ \\
\hline TNF- $\alpha$ & 13.04 & $<0.0001$ \\
PAI-1 & 0.23 & 0.004 \\
TPA & 0.15 & $<0.0001$ \\
\hline
\end{tabular}

TNF- $\alpha$ : tumor necrosis factor $\alpha$; PAI-1: plasminogen activator inhibitor-1; TPA: tissue plasminogen activator.

plasma levels of IL1- $\beta$, IL-6, TNF- $\alpha$, E-Selectin, P-Selectin, V-CAM-1 and ICAM-1.

Immuno-inflammatory variables in relation to TOAST stroke subtypes are shown in Table 3.

Patients with ischemic stroke classified as cardioembolic (CEI) showed, compared to other subtypes, significantly higher median plasma levels of TNF- $\alpha$ [38.5 (22.2-46); $p<0.0001]$, IL-6 [11 (5.5-19). $p=0.0029]$, IL-1 $\beta$ [11.5 (8-13); $p<0.0001]$, whereas plasma levels of Von Willebrand factor in patients with cardioembolic subtype were higher compared to other subtypes but were only near statistical significance [10 $(5-12)(p=0.0053)$ (Table 3$)$.

Furthermore, stroke patients classified as lacunar showed, compared to other subtypes, significantly lower median plasma levels of TNF- $\alpha$ [19.4 (9-23) pg/mL; $p<0.0001]$; IL-6 [(4 (2-9) pg/mL; $p=0.0029)$ ], IL-1 $\beta$ [6 (10-3) $p<0.0001)$ ], whereas plasma levels of Von Willebrand factor in [6 (3-8) ng/mL] patients with lacunar subtype were lower compared to other subtypes, but were only nearly significant statistically (Table 3 ).

Infarct sites at neuroimaging findings (brain CT or MRI) in relation to TOAST diagnostic subtype are shown in Table 4.

Among the clinical, laboratory and immuno-inflammatory variables analyzed in our patients with acute ischemic stroke, TNF- $\alpha, P A I-1$ and TPA on bivariate logistic regression were highly correlated to stroke diagnosis. These variables include one immuno-inflammatory variable $(\mathrm{TNF}-\alpha)(P<0.0001)$ and two thrombotic-fibrinolytic variables (PAI-1 and TPA) $(p=0.004$; $p<0.0001$ ) (Table 5).

Among the other variables maintained in the final model after exclusion of TNF- $\alpha$, PAI-1 and TPA, IL1 $\beta$ (OR: 1.77; 95\% CI:
Table 6

Multivariate logistic regression about relationship between clinical and immunoinflammatory variables and outcome variable (stroke)

\begin{tabular}{lllll}
\hline & Odds ratio & $95 \% \mathrm{CI}$ & $b$ & $p$ \\
\hline IL-1 $\beta$ & 1.79 & $1.51-2.32$ & 0.57 & $<0.0001$ \\
IL6 & 1.04 & $0.95-1.11$ & 0.009 & 0.75 \\
E-selectin & 1.98 & $1.48-2.68$ & 0.67 & 0.0001 \\
VICAM-1 & 1.19 & $1.04-1.32$ & 0.16 & 0.009 \\
ICAM-1-1 & 1.24 & $1.12-1,39$ & 0.25 & 0.0001 \\
vWF & 1.85 & $1.49-2.48$ & 0.62 & 0.0001 \\
Neut & 1.04 & $0.89-1.10$ & 0.02 & 0.0028 \\
Previous stroke & 0.89 & $0.75-1.02$ & 0.004 & 0.68 \\
Previous CAD & 0.77 & $0.63-0.95$ & 0.003 & 0.80 \\
Previous PAD & 0.78 & $0.55-0.95$ & 0.005 & 0.88 \\
\hline
\end{tabular}

IL-1 $\beta$ : interleukin-1- $\beta$; IL6: interleukin-6; V-CAM-1: vascular cell adhesion molecule-1; ICAM-1: intercellular adhesion molecule-1; vWF: von Willebrand factor; neut: neutrophil count. CAD: coronary artery disease; PAD: peripheral artery disease.

1.41-2.22), Selectin-E (OR: 1.96; 95\% CI: 1.38-2) vWF (OR: 1.83; $95 \%$ CI: 1.41-2.38), were significantly associated with acute ischemic stroke diagnosis, whereas IL-6, VICAM-1, ICAM-1 and neutrophil percentage showed only a slight or no association with stroke diagnosis (see Table 6). Previous stroke, CAD and PAD were not associated with acute ischemic stroke diagnosis.

To provide a further demonstration of diagnostic utility for the three markers that at bivariate analysis showed the highest correlation with stroke diagnosis we developed a multivariable logistic regression model, again as described above. The continuous values of TNF- $\alpha$, PAI-1 and TPA showed a significant positive predictive value (PPV), likelihood ratio (see Table 7), with an area under the ROC curve of $98.6 \%, 97.1 \%$ and $99.9 \%$, respectively (see Table 7 ).

\section{Discussion}

The aim of the present study was to assess the potential diagnostic utility of blood-borne protein biomarkers in predicting acute stroke within $72 \mathrm{~h}$ of symptom onset.

We showed that one immuno-inflammatory variable (TNF- $\alpha$ ) and two thrombotic/fibrinolytic (PAI-1 and TPA) variables were significantly associated with stroke diagnosis.

Table 7

Diagnostic power of TNF- $\alpha$, PAI-1 and TPA

\begin{tabular}{|c|c|c|c|}
\hline & TNF- $\alpha$ & TPA & PAI-1 \\
\hline Sensitivity (\%) & 93.33 & 8.67 & 99.17 \\
\hline Specificity (\%) & 96.75 & 86.99 & 99.19 \\
\hline PPV (\%) & 96.55 & 86.67 & 99.17 \\
\hline NPV (\%) & 93.7 & 86.99 & 99.19 \\
\hline LR (+ve) $(95 \% \mathrm{CI})$ & $28.7(11.55-73.46)$ & $6.662(4.28-10.66)$ & $121.975(22.22-690.31)$ \\
\hline $\mathrm{LR}(-\mathrm{ve})(95 \% \mathrm{CI})$ & $0.068(0.035-0.130)$ & $0.153(0.095-0.237)$ & $0.008(0.001-0.0460)$ \\
\hline 'Near' cut-off at max (sens + spec) & 0.73 & 0.23 & 0.18 \\
\hline Area under ROC curve & 0.986 & 0.971 & 0.999 \\
\hline
\end{tabular}

PPV: positive predictive value; NPV negative predictive value; LR (+ve): positive likelihood ratio; LR (-ve): negative likelihood ratio; ROC curve: receiver operating characteristic curve. 
Pathophysiology of neuronal damage in ischemic stroke is based on a ischemic cascade involving inflammatory mechanisms with cytokine activation, adhesion molecule and selectin involvement, endothelial dyfunction and prothrombotic state. Knowledge of the molecules participating in this pathogenic cascade causing neuronal damage probably represents a chance to recognize possible serum markers of ischemic stroke useful to a diagnostic algorithm of acute ischemic stroke. Increases of the proinflammatory cytokines interleukin-1 $\beta$, IL- 6 and TNF- $\alpha$ have been detected in the ischemic cortex $1 \mathrm{~h}$ after middle cerebral artery (MCA) occlusion in experimental models of brain ischemia [8], and several studies have reported elevations of these proinflammatory cytokines in peripheral blood as well as in cerebrospinal fluid in patients with ischemic stroke [15-20]. Nevertheless, pathogenesis of brain ischemia involves both immuno-inflammatory and thrombotic mechanisms, so a good diagnostic panel for ischemic stroke should include a representative biomarker of both these pathogenic aspects. On this basis, in addition to cytokines, adhesion molecules and selectins we also evaluated TPA/PAI plasma levels and vWF plasma levels as marker of thrombotic/fibrinolytic mechanisms and endothelial dysfunction in order to encompass the three main aspects of brain ischemia pathogenesis: endothelial dysfunction, thrombosis and inflammation.

In our study TNF- $\alpha$, PAI- 1 and TPA were significantly associated with ischemic stroke diagnosis.

Plasminogen activator inhibitor type 1 (PAI-1), which forms a complex with tissue-type plasminogen activator (tPA), is a strong inhibitor of fibrinolysis [21]. Until recently, PAI-1 was best known for its antifibrinolytic properties. Nevertheless, Hoekstra et al. [22] reported that elevated plasma PAI-1 is a strong risk indicator for stroke at old age [21-27].

The mechanism that relates plasminogen activation to cerebrovascular disease probably reflects a non-fibrinolytic function of plasmin. Increased plasminogen activation may lead to increased laminin degradation in extracellular brain tissue and, hence, a reduced brain resistance to ischemic damage [28]. Nevertheless, a possible role of PAI- 1 as acute phase molecule is also plausible [29].

A diagnostic marker of ischemic cerebral cascade could also be useful to monitor clinical course and the possible clinical worsening of patients with ischemic stroke. Quickly distinguishing stroke from non-stroke represents a more intriguing possibility to apply to dubious cases prior to the imaging techniques as brain CT or MRI even in the absence of a clear neurological symptomatology.

In our study we have tested the possible predictive power of a series of molecules involved in the inflammatory mechanism of neuronal ischemic damage. Although their clinical applicability could appear unrealistic for the healthcare expenditure, our finding underlines the diagnostic role of TNF- $\alpha$, PAI- 1 and TPA and this finding could suggest to limit to these three immuno-inflammatory and thrombotic/fibrinolytic markers as a possible employment in acute setting.

Possible limitations of the study could be the fact that we have not evaluated neurobiochemical markers such as S100B, NSE or glial fibrillary acidic protein (GFAPI). S100B, however, is expressed not only in brain tissue but also in a variety of other cell types, under both physiological and pathological conditions. Expression of protein S-100 $\beta$ has been observed in white and brown fat, skin and skeletal muscle tissue, melanoma or glioblastoma cells [30-32]. Moreover, S-100B expression in the latter conditions was far below the activity measured after acute central nervous system disorders and the brain specificity of S-100B release was questioned by a number of investigators, and specificity of NSE and CFAP in ischemic stroke is not fully demonstrated.
Another limitation is that our study is an observational study that can only in part suggest whether the biomarkers under discussion might help to identify such patients at an early, prehospital stage and this extremely important point requires future recognition and discussion.

Moreover, our findings are based upon a cohort of patients in whom the diagnosis is already made owing to the fact that the aim of our study is to evaluate the association between some laboratory variables and known stroke diagnosis, but future studies should evaluate the diagnostic role of these biomarkers in patients with suspected ischemic stroke, to evaluate the power of the variables we reported as strictly associated with stroke diagnosis in acute stroke patients. An interesting question is to evaluate whether these observations differ from those in patients with hemorrhagic stroke or alternative acute or chronic neurological conditions.

Only point measurement could be another limitation of the study since repeated measures may be necessary to define the variance as well as the predictive nature of a biomarker [33], but in support of our findings, several studies previously reported the predictive value of only point measurement of inflammatory biomarkers in atrial fibrillation [34] and other clinical conditions such as congestive heart failure [35] acute myocardial infarction [36] and hypertension [37].

The goal of evaluating the diagnostic role of immunoinflammatory and thrombotic-fibrinolytic markers up to $72 \mathrm{~h}$ after symptom onset could further support our only point evaluation.

Lynch et al. [6] reported that four markers are highly correlated with stroke at 6 and $24 \mathrm{~h}$. These included two markers of inflammation and one marker of thrombosis. Furthermore, the extended time evolution of TNF- $\alpha$ response in humans, peaking at 2-3 days, sustain our choice to evaluate biomarker plasma levels within $72 \mathrm{~h}$ of symptom onset.

Although there was a demand for neurobiochemical markers, to date immuno-inflammatory and hemostatic markers could represent the only possible markers of atherothrombotic mechanisms with possible diagnostic implications in acute ischemic stroke setting.

\section{References}

[1] Ford ES. Giles WH Serum C-reactive protein and self reported stroke: findings from the Third National Health and Nutrition Examination Survey. Arterioscler Thromb Vasc Biol 2000;20:1052-6.

[2] Gussekloo J, Schaap MC, Frolich M, Blauw GJ, Westendorp RG. C-reactive protein is a strong but nonspecific risk factor of fatal stroke in elderly persons. Arterioscl Thromb Vasc Biol 2000;20:1047-51.

[3] Clark WM. Cytokines and reperfusion injury. Neurology 1997;4(suppl. 4):14.

[4] Sarainen T, Carpen O, Karjialainen ML, et al. Evolution of cerebral tumor necrosis factor-a production during human ischemic stroke. Stroke 2001;32:1750-8.

[5] Licata G, Tuttolomondo A Corrao S, et al. Immunoinflammatory activation during the acute phase of lacunar and non-lacunar ischemic stroke: association with time of onset and diabetic state. Int J Immunopathol Pharmacol 2006;19(3 (July-September)):639-46.

[6] Lynch JR, Blessing R, White WD, Grecott HP, Newman MF, Laskowitz DT. Novel diagnostic test for acute stroke. Stroke 2004;35:57-63.

[7] Kim JS, Yoon SS, Kim YH, Ryu JS. Serial measurement of interleukin-6, transforming growth factor-beta, and S-100 protein in patients with acute stroke. Stroke 1996;27:1553-7.

[8] Beamer NB, Coull BM, Clark WM, Hazel JS, Silberger JR. Interleukin-6 and interleukin-1 receptor antagonist in acute stroke. Ann Neurol 1995;37:800-5.

[9] Perini F, Morra M, Alecci M, Galloni E, Marchi M, Toso V. Temporal profile of serum anti-inflammatory and pro-inflammatory interleukins in acute ischemic stroke patients. Neurol Sci 2001;22:289-969.

[10] Vila N, Castillo J, Davalos A, Chamorro A. Proinflammatory cytokines and early neurological worsening in ischemic stroke. Stroke 2000;31:2325-9.

[11] Fon EA, Mackey A, Cote R, et al. Hemostatic markers in acute transient ischemic attacks. Stroke 1994;25:282-6.

[12] Takano K, Yamaguchi T, Uchida K. Markers of a hypercoagulable state following acute ischemic stroke. Stroke 1992;23:194-8.

[13] Sato M, Suzuki A, Nagata K, Uchiyama S. Increased von Willebrand factor in acute stroke patients with atrial fibrillation. J Stroke Cerebrovasc Dis 2006 Jan;15(1):1-7. 
[14] Hosmer DW, Lemeshow S. Applied logistic regression. 2nd ed. John Wiley \& Son, Inc.; 2000. pp. 375.

[15] Efron B, Tibshirani R. Improvements on cross-validation: the bootstrap method. J Am Stat Assoc 1997;92:548-60.

[16] Yamasaki Y, Matsuura N, Shozuhara H, Omodera H, Itoyama Y, Kogure K. Interleukin-1 as a pathogenetic mediator of ischemic brain damage in rats. Stroke 1995;26:676-81.

[17] Beamer NB, Coull BM, Clark WM, Hazel JS, Silberger JR. Interleukin-6 and interleukin 1-receptor antagonist in acute stroke. Ann Neurol 1995;37:800-4.

[18] Fassbender K, Rossol S, Kammer T, et al. Proinflammatory cytokines in serum of patients with acute cerebral ischemia: kinetics of secretion and relation to the extent of brain damage and outcome of disease. J Neurol Sci 1994;122:135-9.

[19] Tarkowski E, Rosengren L, Bloomstrand C, et al. Early intrathecal produc tion of interleukin- 6 predicts the volume of brain lesion in stroke. Stroke 1995;26:1393-8.

[20] Farrarese C, Mascarucci P, Zoia C, et al. Increased cytokines release from peripheral blood cells after acute stroke. J Cereb Blood Flow Metab 1999;19:1004-9.

[21] Kohler HP, Grant PJ. Plasminogen-activator inhibitor type 1 and coronary artery disease. N Engl J Med 2000;342:1792-801.

22] Hoekstra T, Geleijnse JM, Kluft C, Giltay EJ, Kok FJ, Schouten EG. 4G/4G genotype of PAI-1 gene is associated with reduced risk of stroke in elderly. Stroke 2003;34:2822-9.

[23] Strickland S. Tissue plasminogen activator in nervous system function and dysfunction. Thromb Haemost 2001;86:138-43.

[24] Luttun A, Lupu F, Storkebaum E, et al. Lack of plasminogen activator inhibitor-1 promotes growth and abnormal matrix remodeling of advanced atherosclerotic plaques in apolipoprotein E-deficient mice. Arterioscler Thromb Vasc Bio 2002;22:499-505.

25] Heymans S, Luttun A, Nuyens D, et al. Inhibition of plasminogen activators or matrix metalloproteinases prevents cardiac rupture but impairs therapeutic angiogenesis and causes cardiac failure. Nat Med 1999;5:1135-42.

[26] De Waard V, Arkenbout EK, Carmeliet P, et al. Plasminogen activator inhibitor 1 and vitronectin protect against stenosis in a murine carotid artery ligation model. Arterioscler Thromb Vasc Biol 2002:22.

[27] Johansson L, Jansson JH, Boman K, et al. Tissue plasminogen activator plasminogen activator inhibitor-1, and tissue plasminogen activator/plasminogen activator inhibitor-1 complex as risk factors for the development of a first stroke. Stroke 2000;31:26-32.

[28] Chen ZL, Strickland S. Neuronal death in the hippocampus is promoted by plasmin-catalyzed degradation of laminin. Cell 1997;91:917-25.

[29] Dong JS, Fujii D, Goto T, et al. Increased expression of plasminogen activator inhibitor- 1 by mediators of the acute phase response: a potential progenitor of vasculopathy in hypertensives. Hypertens Res 2003;26:723.

[30] Zimmer DB, Cornwall EH, Landar A, Song W. The S100 protein family: history, function, and expression. Brain Res Bull 1995;37:417-29.

[31] Hu J, Ferreira A, van Eldik LJ. S100B induces neuronal cell death through nitric oxide release from astrocytes. J Neurochem 1997;69:2294-301.

[32] McAdory BS, van Eldik LJ, Norden JJ. S100B, a neurotrophic protein that modulates neuronal protein phosphorylation, is upregulated during esioninduced collateral sprouting and reactive synaptogenesis. Brain Res 1998;813: 211-7.

[33] MacFadyen RJ. Intraindividual temporal variance of biomarkers and the definition of individualized prognosis in cardiovascular patients. J Hum Hypertens 2006 Jun;20(6):383-6.

[34] Conway DS, Buggins P, Hughes E, Lip GY. Prognostic significance of raised plasma levels of interleukin-6 and C-reactive protein in atrial fibrillation. Am Heart J 2004 Sep;148(3):462-6.

[35] Chin BS, Blann AD, Gibbs CR, Chung NA, Conway DG, Lip GY. Prognostic value of interleukin-6, plasma viscosity, fibrinogen, von Willebrand factor, tissue factor and vascular endothelial growth factor levels in congestive heart failure. Eur J Clin Invest 2003 Nov;33(11):941-8.

[36] Valgimigli M, Ceconi C, Malagutti P, et al. Tumor necrosis factor-alpha receptor 1 is a major predictor of mortality and new-onset heart failure in patients with acute myocardial infarction: the Cytokine-Activation and Long-Term Prognosis in Myocardial Infarction (C-ALPHA) study. Circulation 2005 Feb 22;111(7):863-70.

[37] Varughese GI, Patel JV, Tomson J, Blann AD, Hughes EA, Lip GY. Prognostic value of plasma soluble P-selectin and von Willebrand factor as indices of platelet activation and endothelial damage/dysfunction in high-risk patients with hypertension: a sub-study of the Anglo-Scandinavian Cardiac Outcomes Trial. J Intern Med 2007 Apr;261(4):384-91. 